\title{
Effect Of High And Low Resilience On Coping And Depressive Symptoms Among Caregivers Of Psychiatric Patients Versus Caregivers Of Patients Suffering from Chronic Medical Illness
}

\author{
Pinkala Singh ${ }^{1}$ \\ ${ }^{1}$ Post Graduate Student, Department of Psychology, Smt. Maniben Nanavati Women's College, \\ Mumbai.
}

\begin{abstract}
The purpose of this study was to investigate coping and depressive symptoms among resilient caregivers of psychiatric patients versus caregivers of patients suffering from chronic medical illness. The sample comprised of ninety two caregivers (forty one caregivers of psychiatric patients and forty one caregivers of patients suffering from chronic medical illness) who will be assessed on Brief resilience scale, Brief Coping Scale and Becks Depression Inventory. Results indicate that Caregivers with higher level of resilience had lower depressive symptoms and high coping, while dealing patients with psychiatric and chronic medical illness. Also type of illness was not a significant predictor for differences in coping and depressive symptoms.
\end{abstract}

Key words - Resilience, Caregivers, Depressive symptoms, Psychiatric IIIness, Chronic Medical Illness.

\section{INTRODUCTION}

In India, neuropsychiatric disorders are estimated to contribute to $11.6 \%$ of the global burden of disease [1]. Looking at the growing estimates of illness the one most affected is of this is the patient and the relatives who take care of them. In India, the family is involved in the care of the patients in the hospital from the first day itself. A family member is sitting by the bed in the intensive care unit and throughout the entire hospitalization. Families are an integral part of the care system for persons with a chronic mental illness [2]. Psychiatric patients need assistance or supervision in their daily activities and this often places a major burden on their caregivers, thereby placing the caregiver at a great risk of mental, emotional and a practical impact like physical health problems on the caregiver [3-5].

The burden upon caregivers for a mentally ill patient living at home was first acknowledged by Grad and Sainbury in the early 1960s [6]. The most common mental health consequences identified are depression, anxiety and burnout which occur when a caregiver slips beyond exhaustion or depression. Studies showed that caregivers reported burden in different areas including effects on family functioning, social isolation, and financial problems. The financial constraints and the lack of social 
support can cause family caregivers to feel helpless and leave them little time to appropriately complete personal responsibilities [7]. In addition, caregivers often feel a moral obligation to continue to care for relatives at home. This obligation is influenced by cultural beliefs, gender role, and perceptions of institutional constraints on care [8].

Caregivers in order to take care of the stressful situation such as taking care of patient with chronic physical or psychological illness may experience emotional, social or physical imbalance affecting the family functioning. In analysing the resilience of a caregiver, the following assumptions are made: adversity or chronic stressors invite challenge, creating more possibilities, and people (caregivers) have intrinsic characteristics and capacities to heal themselves and are capable of adapting to adversity [9].

The relationship between depressive symptoms, coping and resilience are of great importance identifying the coping strategies utilized by resilient individuals may predict which clients are at risk of depression. The purpose of the present study is to examine the effects of resilience on coping levels and levels of depression among family member caregivers of psychiatric patients versus family member caregivers of physically ill patients without any psychiatric illness. The independent variables are high and low resilience in caregivers of individuals suffering from psychiatric illness and chronic medical illness and outcome as caregivers' coping and depressive symptoms.

\section{METHODOLOGY}

An ex-post facto research design was used because the investigation starts after the fact has occurred without interference from the researcher. The sampling technique that was used was purposive and snowballing where a specific group is selected and a snowball sample is achieved by asking a participant to suggest someone else who might be willing or appropriate for the study. Snowball samples are particularly useful in hard-to-track populations, such as truants, drug users, etc. Each participant received researcher developed demographic detail which were filled in a semistructured questionnaire, Brief Resilience Scale [10], Brief Coping Scale [11] and Beck Depression Inventory [12]. These were administered in order to assess resilience, coping and depression among caregivers. Demographic details were designed to collect descriptive information about the personal characteristics of the participants in order to describe the sample.

Caregivers aged more than 20 years who were staying with the patient since the onset of illness were included in the study. The caregivers aged less than 20 years were excluded from the study. They need to be a family member living in the same house and taking care of the patient. Each caregiver included was explained about the study and informed consent was taken. Patient with only high and low resilience on Brief Resilience scale were selected for the study. Data were coded as numbers and entered into a computer database program. The obtained data were than analyzed with descriptive statistics on windows excel 2007 and ANOVA was calculated on computerized software. Tukey HSD post hoc test was performed after ANOVA to identify any specific differences for significant multivariate tests.

\section{RESULTS}

Participants in the study were 92 family members mostly involved in taking care of patients with severe psychiatric and physical illness. They did 
not provide care in exchange of financial compensation. The psychiatric sample included schizophrenia (19), mood disorder (19), substance abuse (8) and mental retardation with psychosis (5) that were diagnosed as per the guidelines given in DSM-IV-TR [13] and caregivers of chronic medical illnesses include patients suffering from cardiovascular disease (31), Cancer(3), Kidney (2) and neurological disorder (5). For every patient, a single caregiver was considered. The sample consisted of 92 caregivers, 57 were female and 35 male, with a mean age of 39 years, and the ages ranged from 20 to 65 . The mean age for psychiatric patients (34.98 years) was lower than the mean age for patient suffering from chronic physical illness (50.7 years). Among 92 participants, $12(13.04 \%)$ people are illiterate, $28(30.43 \%)$ had got below tenth standard of formal education, 51 $(55.43 \%)$ has studied tenth standard or are graduate. Also among 92 people, 15 were unmarried and 76 were married. There were 44 people with income below one lakh per annum and 48 below 5 lakh per annum while employment percentage was $50 \%$ for unemployed and employed.

A two-way factorial ANOVA for Independent sample was performed to compare the means of caregivers and resilience with depression. ANOVA analysis (Table 1) revealed a significant difference between the resilience and depression ( $\mathrm{df}=1$, $\mathrm{F}=21, \mathrm{p}<0.0001)$. The absolute difference between the means $(4.13,9.61)$ required for significance at HSD 0.5 level is higher than the critical value (2.3) for the Tukey HSD test as shown in table 2 . This indicated that caregivers with higher level of resilience would have lower depressive symptoms, in dealing with both psychiatric patients and patients with chronic medical illness.

Table - 1 Two Way ANOVA Summary for Depressive Symptom

\begin{tabular}{|c|c|c|c|c|c|}
\hline Source & SS & df & MS & F & P \\
\hline Caregivers & 21.6 & 1 & 21.6 & 0.7 & 0.4051 \\
\hline Resilience & 659.11 & 1 & 659.11 & 21.48 & $<.0001$ \\
\hline r x c & 19.02 & 1 & 19.02 & 0.62 & 0.4332 \\
\hline Error & 2700.76 & 88 & 30.69 & \multicolumn{2}{|l}{} \\
\cline { 1 - 4 } Total & 3433.61 & 91 & \multicolumn{1}{|l}{}
\end{tabular}

Table 2 - Critical Value for Tukey HSD of Depressive symptom

\begin{tabular}{|c|c|c|}
\hline & HSD [.05] & HSD [.01] \\
\hline CAREGIVER [2] & 2.31 & 3.06 \\
\hline RESILIENCE [2] & 2.3 & 3.04 \\
\hline Cells [4] & 4.31 & 5.27 \\
\hline
\end{tabular}

ANOVA analysis (Table 3 ) revealed a significant difference between the resilience and coping ( $\mathrm{df}=1, \mathrm{~F}=7, \mathrm{p}=0.007$ ). The absolute difference between the mean $(40.30,36.85)$ required for significance at HSD 0.5 level is higher than the critical value (2.69) for the Tukey HSD test shown in table 4. Analysis of variance was performed on caregivers of psychiatric patients versus chronic medical illness and resilience across two variables - depressive symptoms and coping. ANOVA Analyses (Table 1) showed that there were no significant differences amongst caregivers of 
psychiatric patients with higher resilience and depressive symptoms ( $d f=1, F=0.62, p$ $=0.43$ ). This indicated that caregivers of psychiatrically ill patients with higher resilience would not have the lowest depressive symptoms. Also analysis showed that there were no significant differences among caregivers of psychiatric patients with higher resilience and coping ( $\mathrm{df}=1, \mathrm{~F}=2.77, \mathrm{p}=0.12$ ). This indicated that caregivers of physically ill patients with higher resilience would not have the highest coping levels. ANOVA revealed that there was no statistical significant difference between caregivers of psychiatric patients and chronic medically ill patients with regards to coping $(p=0.18$ ) or depressive symptoms $(p=0.40)$.

Table 3 - Two Way ANOVA Summary for Coping

\begin{tabular}{|c|c|c|c|c|c|}
\hline Source & SS & df & MS & F & P \\
\hline Caregivers & 75.61 & 1 & 75.61 & 1.8 & 0.1832 \\
\hline Resilience & 312.5 & 1 & 312.5 & 7.44 & 0.0077 \\
\hline r x c & 102.52 & 1 & 102.52 & 2.44 & 0.1219 \\
\hline Error & 3695.82 & 88 & 42 & \multicolumn{1}{|l}{} \\
\hline Total & 4156.73 & 91 & \multicolumn{1}{|l}{}
\end{tabular}

Table 4 - Critical Values for the Tukey HSD Test of Coping

\begin{tabular}{|c|c|c|}
\hline & HSD [.05] & HSD [.01] \\
\hline CAREGIVER [2] & 2.7 & 3.58 \\
\hline RESILIENCE [2] & 2.69 & 3.56 \\
\hline Cells [4] & 5.05 & 6.17 \\
\hline
\end{tabular}

\section{DISCUSSION}

Females are the traditional caregivers for patients with chronic illnesses as reported in many studies and also shown in this study as well. The patients' mean ages especially for psychiatric patient showed that they were within the productive age group, which implies a loss of productivity that in turn has many economic implications, not only for caregivers and patients. While undertaking the interview it was revealed that the caregiver were facing these problems

As caregivers had to take care of the patient they did not spend time on self care. Family members are affected more when the patient was hospitalized frequently. There was lack of support from others during the illness. Most of the time caregivers were victims of patient's violence. This also contributed to their ill health. Most of the patients who had come for treatment belonged to lower economic group $(47.83 \%)$ which was another reason of concern for the caregiver as they were facing problems due to expenses in different areas like drug therapy, charges for hospitalization and transportation. In some cases, stress seemed to be associated with a triple shift i.e. job, household duties, and care for a patient. It got worst when the patient was hospitalized. This was especially true for male caregivers. 
This study hypothesized that there would be a significant difference between resilience and depressive symptoms, such that changes in the level of resilience would affect depressive symptoms. A convenient sample of 92 caregivers ( 51 caregivers of psychiatric patients and 41 patients with chronic medical illness), ranging from 20 to 65 years were selected for the study. Researchers [14] have found that caregiver burden scores in the caregivers of psychiatric patients were significantly higher than that of chronic medical illness $(p<0.0001)$ and that the caregiver burden was found to increase with the duration of illness as well as with the age of caregiver. The study revealed that there is no significant difference in depressive symptom $(p=0.4051)$ and coping $(p=$ 0.1832 ) among the caregivers of psychological illness and chronic medical illness. These results indicate that even if both psychiatric illness and chronic medical illness are two different categories of illnesses and the amount of caregiver burden being different the amount of coping that the patient used and depressive symptoms in the patient were not different. The reason for this may be that the level of stress was similar in both the groups.

This has been shown in other research papers as well [3-5, 15] that patients need assistance or supervision in their daily activities and this often places a major burden on their caregivers, thereby placing the caregiver at a greater risk of mental, emotional and a physical impact like physical health problems on the caregiver. The present study further explores the effect of resilience on depressive symptoms amongst the caregivers. There was a significant difference between resilience and depressive symptoms. The result of this study was in agreement with those of other similar other studies [16] and that higher levels of resilience were associated with lower levels of self-reported depression or depressive symptoms. Resilience is seen in relation to positive age appropriate development (such as positive peer relationships), resources and adaptive capabilities, and not just on the absence of symptoms or risks. The presence of certain assets rather the mere absence of risk is one way in which resilience may be conferred [17]. As risk levels increase, so resilience levels need to increase to counter their effect. The effect of resilience on coping among caregivers was also found significant in congruence with previous research [18] indicating that high resilience lead to high coping. Though here coping is not been categorized as emotion-focused or problem-solving, an overall view is considered as has been indicated [19] such that problem-focused and emotion focused coping complement each other.

Lastly our results show that a negative correlation between depressive symptoms and coping, Where high coping leads to low depressive symptoms. Research has shown that coping had led to reduced depressive symptoms [20]. In the study by Perlick, [20] it was found that social support and avoidance coping accounted for $63 \%$ of the relationship between caregiver stigma and depression. Patient's clinical and functional status was evaluated within 30 days of the caregiver assessment. Perceived stigma was positively associated with caregiver depressive symptoms, controlling for patient status and socio-demographic factors.

The present study will add to the scientific understanding of perceived ethnic discrimination, its impact on mental health, and the manners in which individuals cope with it. However certain limitations do exist, and should be taken into consideration. The first limitation is that the sample size is small, and duration of the study is too small to consider effective research. Also, the study did not separate groups by gender, ethnicity, or age, and thus does not reflect any intergroup or intragroup 
differences that may exist. Furthermore the sample used for data collection is a unique subset of the emerging adult population (caregivers were selected from Mumbai and most of them from same hospital), and for this reason may not generalize to the larger population of early adults. Hence, future studies may want to use a larger, more representative and diverse sample.

\section{CONCLUSIONS}

This study provides the rationale for including families in the assessment and treatment of patients and provides guidelines for physicians and other clinicians when working with family members of patients with chronic physical and mental illness. For the caregiver, low perceived stress and good coping skills result in the most reward and least burden. The caregivers benefit when the physician acknowledges their burden, supports their need for self care and helps set appropriate limits with the patient.

\section{REFERENCES}

1. World Health Organization. Country health profile. Retrieved from website: http://www.who.int/gho/countries/ind.pdf ; 2008.

2. Shankar R, Menon S. Interventions with families of people with schizophrenia. The issues facing a community based rehabilitation center in India. Psychosoc Rehabil J 1993;15:8591.

3. Ampalam P, Gunturu S, Padma V. A comparative study of caregiver burden in psychiatric illness and chronic medical illness. Indian J Psychiatry 2012;54:239-243.

4. Chakrabarti S, Gill S. Coping and its correlates among caregivers of patients with bipolar disorder: a preliminary study. Bipolar Disord 2002;4(1):50-60.

5. Provencher $\mathrm{HL}$. Objective burden in primary caregivers of persons with chronic schizophrenia. J Psychiatr Ment Health Nursing 1996;3:181-187.

6. Krupnik Y, Pilling S, Killaspy H, Dalton J. A study of family contact with clients and staff of community mental health teams. Psychiatric Bull 2005;29:174-176.

7. Grunfeld E, Coyle D, Whelan T, Clinch J, Reyno L, Earle CC, Glossop R. Family caregiver burden: Results of a longitudinal study of breast cancer patients and their principal caregivers. Can Med Assoc J 2004;170:1795-1801.

8. Sellappah S, Kristjanson L, Maltby $\mathrm{H}$. Cancer in West $\neg$ ern Australian Indian families: Concerns and coping strategies. Int J Palliative Nurs 2001;7:588-596.

9. Fang-Yi L, Jiin-Ru R, Tzu-Ying L. Resilience among caregivers of children with chronic conditions: a concept analysis. J Multidisc Healthcare 2013;6:323-333.

10. Smith BW, Dalen J, Wiggins K, Tooley E, Christopher P, Bernard J. The brief resilience scale : assessing the ability to bounce back. Int J Behav Med 2008;15:194-200.

11. Carver CS. You want to measure coping but your protocol is too long : consider the brief coping scale. Int J Behav Med 2007;4:92-100.

12. Beck AT, Steer RA, Carbin MG. Psychometric properties of the Beck Depression Inventory : twenty five years of evaluation. Clin Psychol Rev 1988;8:77-100.

13. American Psychiatric Association. Diagnostic and Statistical Manual for the Classification of Psychiatric Disorders $-4^{\text {th }}$ edition - text revised (DSM-IVTR). American Psychiatric Publishing ; 2000.

14. Ampalam P, Deepthi R, Vadaparty P. Schizophrenia - insight, depression : a correlation study. Indian J Psychol Med 2012;34:44-48.

15. Savage S, Bailey $S$. The impact of caring on caregivers' mental health: a review of the literature. Austr Health Rev 2004;27:111-117.

16. Spies G, Seedat S. Depression and resilience in women with HIV and early life stress: does trauma play a mediating role? A cross-sectional study. BMJ Open 2014;4:e004200. 
17. Masten AS. Resilience in developing systems : progress and promise as the fourth wave rises. Dev Psychopath 2007;19:921-930.

18. Cherkil S. Coping styles, stress tolerance and well being and their correlations in spouses of the mentall ill. Indian J Psychol Med 2010;32:99-103.

19. Folkman S. Personal control and stress and coping processes: A theoretical analysis. J Personal Soc Psychol 1984;46:839-852.

20. Perlick DA, Miklowitz DJ, Link BJ, Struening E, Kaczynski R, Gonzalez J, Manning LN, Wolff $\mathrm{N}$, Rosenheck RA. Perceived stigma and depression among caregivers of patients with bipolar disorder. Br J Psychiatry 2007;190:530-536.

Acknowledgements - Nil

Conflict of Interest - Nil

Funding - Nil 